\title{
Chain models, trees of singular cardinality and dynamic EF-games*
}

\author{
Mirna Džamonja ${ }^{\dagger}$ and \\ Jouko Väänänen
}

\begin{abstract}
Let $\kappa$ be a singular cardinal. Carol Karp's notion of a chain model of size $\kappa$ is defined to be an ordinary model of size $\kappa$ along with a decomposition of it into an increasing union of length $\mathrm{cf}(\kappa)$. With a notion of satisfaction and (chain)-isomorphism such models give an infinitary logic largely mimicking first order logic. In this paper we associate to this logic a notion of a dynamic EFgame which gauges when two chain models are chain-isomorphic. To this game is associated a tree which is a tree of size $\kappa$ with no $\kappa$-branches (even no $\operatorname{cf}(\kappa)$ branches). The measure of how non-isomorphic the models are is reflected by a certain order on these trees, called reduction. We study the collection of trees of size $\kappa$ with no $\kappa$-branches under this notion and prove that when $\operatorname{cf}(\kappa)=\omega$ this collection is rather regular; in particular it has universality number exactly $\kappa^{+}$. Such trees are then used to develop a descriptive set theory of the space ${ }^{\mathrm{cf}(\kappa)} \kappa$.

The main result of the paper gives in the case of $\kappa$ strong limit singular an exact connection between the descriptive set-theoretic complexity of the chain isomorphism orbit of a model, the reduction order on the trees and winning strategies in the corresponding dynamic EF games. In particular we obtain a neat analogue of the notion of Scott watershed from the Scott analysis of countable models.
\end{abstract}

Keywords: trees, singular cardinal, rank.

MSC 2000 Classification: 03E04, 54E99

*Mirna Džamonja thanks EPSRC for their support through an Advanced Fellowship through the early parts of this research. Jouko Väänänen's research was partially supported by grant 40734 of the Academy of Finland.

†School of Mathematics, University of East Anglia, Norwich, NR4 7TJ, UK, M.Dzamonja@uea.ac.uk, http://www.mth.uea.ac.uk/people/md.html

${ }_{\ddagger}^{\ddagger}$ Department of Mathematics, University of Helsinki, Helsinki, Finland, and Institute for Logic, Language and Computation, University of Amsterdam, Netherlands, jouko.vaananen@helsinki.fi, http://www.logic.math.helsinki.fi/people/jouko.vaananen/ 


\section{Introduction}

We shall work with $\kappa$ a singular cardinal of countable cofinality, and our interest is in rooted trees of height and cardinality $\kappa$. We call such trees $\kappa$-Trees (as opposed to the usual $\kappa$-trees, which are also required to have levels of size $<\kappa$ ). In particular, we are interested in $\kappa$-Trees which in addition do not have $\kappa$-branches. We call $\kappa$-Trees with this last property bounded, otherwise a $\kappa$-Tree is said to be unbounded. In analogy with the body of knowledge which is available about $\lambda$-Trees for $\lambda$ regular (see [ToVä]) we study the class of bounded $\kappa$-Trees under the natural notion of reduction, which is simply a strict-order preserving function from one tree to another.

Let us now explain how these considerations have arisen in the context of model theory and descriptive set theory.

A relational structure of size $\lambda$ can be considered as an element of $2^{\lambda}$, which is an insight going back to 1930s, in particular to C. Kuratowski and A. Tarski [KuTa]. This insight was first exploited in the context of countable models. To understand the classification of such models up to isomorphism Ehrenfeucht [Eh], building on the work of Fraïssé [Fr1], [Fr2], introduced a game now known as the EF game. There is an even older method of classification, using the fact that for two fixed countable models the set of all isomorphisms between them is $F_{\delta \sigma}$, and hence the set of pairs of models that are non-isomorphic is co-analytic. It therefore can be covered by $\aleph_{1}$ many Borel sets, by the covering theorem of Lusin-Sierpiński [LuSi]. In fact, the main content of the Ehrenfeucht-Fraïssé Theorem (see e.g. [ChKe]) is that this set,

$$
\{(A, B): A, B \text { countable models and } \exists f: A \cong B\}
$$

is the same set as the set of pairs $(A, B)$ of countable models for which II has a winning strategy in the EF game. By Dana Scott's analysis in the 1960s [Sc] the latter can be seen as the intersection of $\aleph_{1}$ Borel sets arising from ranking the EF game by the countable ordinals. This analysis made it possible to attach to each pair $(A, B)$ of non-isomorphic countable models a rank, called Scott watershed $S(A, B)$, which in this case is an ordinal $\alpha<\omega_{1}$. The rank can be thought of as a clock in the following sense: during the EF game the Nonisomorphism player I has to at every stage go down this clock, starting at $\alpha$ itself, and a condition of winning for I is that he has not run out of time before the actual nonisomorphism has been exposed. This game is called the dynamic $E F$ game of rank $\alpha+1$ and denoted by $E F D_{\alpha+1}$ (the notation comes from [Vä2]). The fact that $S(A, B)=\alpha+1$ means that II wins $E F D_{\alpha}$ (and hence $E F D_{\beta}$ for any $\beta<\alpha$ ), while I wins $E F D_{\alpha+1}$ (and hence $E F D_{\beta}$ for any $\beta>\alpha$ ).

For uncountable models, say of size $\aleph_{1}$, one can generalise the Ehrenfeucht-Fraïssé Theorem by considering games of length $\omega_{1}$. One is then tempted to find the corresponding notion of the Scott watershed. It turns out that it is no longer enough to use the 
ordinals, as the game is transfinite. The right notion this time is that of a tree of size $\aleph_{1}$ with no uncountable branches. The class $\mathcal{T}_{\aleph_{1}}$ of these trees is ordered by reductions, which are strict order-preserving functions. We denote the existence of a reduction from $T$ to $T^{\prime}$ by $T \leq T^{\prime}$. Furthermore, we write $T<T^{\prime}$ if both $T \leq T^{\prime}$ and $T^{\prime} \not \leq T$. If $T \leq T^{\prime}$ then it is easier for II to win $E F D_{T}$ than $E F D_{T^{\prime}}$. Respectively, if $T \leq T^{\prime}$ then it is easier for I to win $E F D_{T^{\prime}}$ than $E F D_{T}$. Finally, if II wins $E F D_{T}$ and I wins $E F D_{T^{\prime}}$, then one can prove $T<T^{\prime}$. This approach was gradually developed in Helsinki in the 1980s, culminating in the paper [HyVä] where the general theory was established, and in particular it was shown that the analogue of the Scott watershed exists also in the uncountable case. Naturally, the importance of $\left(\mathcal{T}_{\aleph_{1}}, \leq\right)$ in this context led to a systematic study of its structural properties (e.g. [MeVä], [ToVä]). Descriptive set theory of the space $2^{\omega_{1}}$ based on $\mathcal{T}_{\aleph_{1}}$ (see again [MeVä]) was subsequently developed. The theory of $\left(\mathcal{T}_{\lambda}, \leq\right)$ for $\lambda$ successor of regular is also quite known, although it is not completely parallel to that of $\left(\mathcal{T}_{\aleph_{1}}, \leq\right)$-see [Vä2]. Juha Oikkonen [Oi] proved in 1991 a covering theorem for a certain infinite quantifier infinitary logic, using trees of singular cardinality. In this paper we develop the structure theory of $\left(\mathcal{T}_{\kappa}, \leq\right)$ for $\kappa$ singular, concentrating especially on $\kappa$ of countable cofinality.

When we move to such cardinals new possibilities open up. Let us look at the infinitary logic $L_{\kappa \kappa}$, assuming that $\kappa$ is singular of cofinality $\omega$. Starting with Carol Karp's Ph.D. thesis in 1959 and in later work (see [Ka]) Karp and others showed that one can develop model theory of $L_{\kappa \kappa}$ based on the concept of a chain model. This was further developed by Michael Makkai [Mak]. A chain model ${ }^{1}$ is an ordinary model $A$ equipped with a presentation of $A$ as a union of a chain $A_{0} \subseteq A_{1} \ldots \subseteq A_{n} \subseteq \ldots$ for $n<\omega$. The chain is not assumed to be elementary. Let us denote such a system as $\left(A_{n}\right)$. The point of chain models is the following modification of the truth definition of $L_{\kappa \kappa}$

$$
\left(A_{n}\right) \models \exists \bar{x} \varphi(\bar{x}) \Longleftrightarrow \text { there are } n<\omega \text { and } \bar{a} \in A_{n} \text { with } A_{n} \models \varphi(\bar{a}),
$$

where $\bar{x}$ is a sequence of length $<\kappa$. If we restrict to chain models, the model theory of $L_{\kappa \kappa}$ is very much like that of $L_{\omega_{1} \omega}$. The fundamental observation of Karp was that the proof of the Completeness Theorem of $L_{\omega_{1} \omega}$ using consistency properties works fine when we have infinite homogeneous strings of quantifiers if we modify truth definition as above in (1). Thus we can also prove undefinability of well order, Craig Interpolation Theorem, Beth Definability Theorem, etc. None of these theorems is true for the classical $L_{\kappa \kappa}$ logic, by the work of Gostanian and Hrbaček ([GoHr]) and Malitz ([Mal]). A discussion involving many of these developments can be found in [Vä1]. Our paper can be seen as an extension of Scott's analysis of countable models to chain models of size $\kappa$. This possibility was announced by C. Karp in [Ka]. Most likely this promising direction would have been continued by her had it not been for her premature death.

\footnotetext{
${ }^{1}$ This is the definition used by C. Karp. We use a somewhat different language, see Definition 1.2.
} 
There are several natural version of EF game for (chain) models of a singular cardinality $\kappa$. We show in Section 1 that the relevant clock trees of these games are trees of height $\kappa$ without $\kappa$-branches. Thus to understand the Scott watershed phenomenon an investigation of the order of such trees under reduction is called for. This is what we set out to accomplish in this paper.

We shall show that $\kappa$-Trees for $\kappa$ as above have properties that make them rather similar to ordinals. The reason for this is that there is a natural notion of rank. Using this notion we can for example show that the universality number of unbounded $\kappa$-Trees under reduction is just $\kappa^{+}$, and that within each rank in $\left[1, \kappa^{+}\right)$it is $\omega$. This is in sharp contrast with the situation of $\lambda$-Trees where $\lambda$ is a regular cardinal. For example for $\lambda=\omega_{1}$ Mekler and Väänänen [MeVä] have established that the universality number for $\lambda$-Trees cannot be computed in ZFC.

Another insight of [MeVä] is the use of $\omega_{1}$-Trees in the descriptive set theory in ${ }^{\omega_{1}} \omega_{1}$. In analogy with that we consider Section 7 the descriptive set theory of ${ }^{\operatorname{cf}(\kappa)} \kappa$ for $\kappa$ singular strong limit. We present a covering theorem for $\Pi_{1}^{1}$ subsets of the topological space ${ }^{\theta} \kappa$ where $\theta=\operatorname{cf}(\kappa)$ and $\kappa$ is singular strong limit. This result was inspired by a theorem of Juha Oikkonen [Oi] from 1991 who proved a covering theorem for an infinitary logic. Our covering theorem and that one from $[\mathrm{MeVä}]$ are obtained by using a corresponding class of trees, in our case the $\kappa$-Trees which we studied in the rest of the paper.

Our notation is standard, in particular, $h_{T}(t)$ denotes the height of an element $t$ in a tree $T$. The order on $T$ is denoted by $\leq_{T}$. If $T$ is clear from the context we may omit it. A tree is said to be normal if for any $t \neq t^{\prime}$ of a limit height, we have $\left\{s: s<_{T} t\right\} \neq\left\{s: s<_{T} t^{\prime}\right\}$. Level $\alpha$ of $T$ is denoted by $\operatorname{lev}_{\alpha}(T)$. If $t \in T$ then $T[\geq t]$ is the set $\{s \in T: s \geq t\}$ equipped with the order inherited from $T$. The following is a crucial definition:

Definition 0.1 A reduction from a tree $T$ into a tree $T^{\prime}$ is a function $f: T \rightarrow T^{\prime}$ such that whenever $x<_{T} y$ in $T$, then $f(x)^{\prime}<_{T} f(y)$. If there is such a reduction we write $T \leq T^{\prime}$.

We shall also make use of the following operator $\sigma^{2}$ defined on the class of trees:

Definition 0.2 If $T$ is a tree then $\sigma T$ is the tree whose elements are strictly $<_{T^{-}}$ increasing sequences of the elements of $T$, ordered by end extension.

The importance of this operator is that it is easily checked that there is no reduction from $\sigma T$ into $T$.

\footnotetext{
${ }^{2}$ The definition of $\sigma$ comes from Kurepa's work on Souslin's hypothesis, see [ToVä] for more.
} 
Most of the trees we consider in this paper will have size and height $\kappa$ for some fixed cardinal $\kappa$, with no restriction on the size of the levels of the tree. We call such trees $\kappa$-Trees, to be distinguished from the usual $\kappa$-trees whose levels are also required to have size $<\kappa$. A $\kappa$-Tree is said to be bounded if it does not have a branch of length $\kappa$. A $\kappa$-Tree that has no branches of length $\mu$ is called a $(\kappa, \mu)$-Tree.

The paper is organised as follows. In section 1 we show how $\kappa$-Trees and games involving them arise naturally in the context of C. Karp's chain models. In section 2 we introduce a rank operation and show that it characterizes bounded $\kappa$-Trees, and then we introduce a game characterisation of ranks. In section 3 we introduce various operations on trees which will allow us to study the structure of the class of $\kappa$-Trees. In $\S 4$ we consider how one can relate the known results about universality of trees at uncountable regular $\theta$, say at $\theta=\aleph_{1}$, to singular cardinals of that cofinality. This shows that the $\mathrm{ZFC}$ results we obtain later in $\S 5$ for cofinality $\omega$ must be special to that cofinality. In $\S 5$ we obtain these ZFC results, allowing us the exact calculation of the universality number of the class of $\kappa$-Trees, even within any given rank. In section 6 we consider the problem of the existence of incomparable trees. Finally, in section 7 we give the promised descriptive set-theory results for the space ${ }^{\mathrm{cf}(\kappa)} \kappa$ for $\kappa$ singular strong limit, and we finish by giving a theorem which in the case of such cardinals connects all of the mentioned notions, giving an exact connection between chain isomorphisms, descriptive set-theoretic complexity and the generalised EF games and trees.

\section{Chain models and $\kappa$-Trees}

To show how $\kappa$-Trees and games involving them arise naturally in the context chain models, we first review the case of ordinary models. Recall the notion of the Scott watershed from the Introduction.

Let $\kappa$ be an infinite cardinal. The original $E F$ game, denoted $E F_{\kappa}(A, B)$, for models $A$ and $B$ of size $\kappa$ is as follows: players play elements of $A \cup B$ for $\kappa$ rounds, and at each round II plays in the model in which I did not play at that round. II wins this game if the end result is a partial isomorphism between $A$ and $B$. Obviously, $A \cong B$ iff $I I$ has a winning strategy in $E F_{\kappa}(A, B)$. Suppose that in addition to the models $A$ and $B$ we are given a tree $T$. The dynamical version $E F D_{T}(A, B)$ of $E F_{\kappa}(A, B)$ is defined by requiring the player $I$ to have the extra task of moving up the tree $T$ at every move, and if he runs out of the space to move, he loses.

Let $\tau$ be the strategy of $I$ in which he systematically lists elements of $A \cup B$. Let $S_{1}$ be the tree of plays of II against $\tau$ where she has not lost yet. Let $K_{1}$ be the tree of winning strategies of $I I$ in shorter versions of $E F_{\kappa}(A, B)$ where we limit the length of the game to be an ordinal $<\kappa$. The ordering of the strategies of $K_{1}$ is canonical: a 
strategy $\rho$ precedes another strategy $\rho^{\prime}$ if $\rho$ is the restriction of $\rho^{\prime}$ to the length of the game in $\rho$.

Lemma 1.1 Assume that $\kappa$ is a cardinal and $A, B$ are models. Then $K_{1} \leq S_{1}$. If $A \neq B$ then the trees $S_{1}$ and $K_{1}$ do not have $\kappa$-branches, and $K_{1} \leq S_{1}$. Moreover, in this case II wins $E F D_{K_{1}}(A, B)$ and $I$ wins $E F D_{\sigma S_{1}}(A, B)$.

Proof. If $\rho \in K_{1}$, define $f(\rho)$ to be the result of applying $\rho$ to $\tau$ - i.e. both players play their strategies. The result is in $S_{1}$. Clearly this function is increasing. Thus $K_{1} \leq S_{1}$. It is an immediate consequence of $A \not B$ that $S_{1}$ and $K_{1}$ do not have $\kappa$-branches. The reason why $I I$ wins $E F D_{K_{1}}(A, B)$ is that the moves of $I$ in $K_{1}$ reveal longer and longer strategies that $I I$ can use against $I$. The reason why $I$ wins $E F D_{\sigma S_{1}}(A, B)$ is that he can submit the sequence of previous moves of $I I$ as his moves in the tree $\sigma S_{1}$.

So we have found upper $\left(\sigma S_{1}\right)$ and lower $\left(K_{1}\right)$ bounds for a Scott watershed. Our cardinal number $\kappa$ can be also singular. However, the trees $S_{1}$ and $K_{1}$ need not be of cardinality $\kappa$. All we can say is that $\left|S_{1}\right| \leq \kappa^{<\kappa}$ and $\left|K_{1}\right| \leq \kappa^{\kappa^{<\kappa}}$, and so in the case of singular cardinal $\kappa$ no cardinal arithmetic assumption may assure us that these trees have size $\kappa$.

We shall now modify the above EF game to take into account further information about the models $A$ and $B$. The further information is that the models are built up from a chain of smaller models.

Definition 1.2 $A$ chain model (of length $\mu$ ) consists of a model $A$ and a decomposition $\left\langle A_{\alpha}: \alpha<\mu\right\rangle$. It is assumed that $A=\bigcup_{\alpha<\mu} A_{\alpha}$, and that $\left\langle A_{\alpha}: \alpha<\mu\right\rangle$ is an increasing sequence of models satisfying $\left|A_{\alpha}\right|<|A|$. A chain isomorphism between $A$ and $B$ is an isomorphism $f: A \rightarrow B$ such that for all $\alpha$ the image of $A_{\alpha}$ is contained in some $B_{\beta}$ and, conversely, the preimage of any $B_{\alpha}$ is contained in some $A_{\beta}$. If there is a chain isomorphism between $A$ and $B$ we write $A \cong{ }_{\text {chain }} A$.

The original language used by Karp to study chain models did not involve trees as clocks, however we can now show that chain models lend themselves to an analysis using exactly the $\kappa$-Trees as considered in this paper.

The most obvious EF game for chain models $A$ and $B$ of size $\kappa$ is the following: The players play elements of $A \cup B$ for $\kappa$ rounds. At any given moment all the played elements have to be contained in some $A_{\alpha} \cup B_{\alpha}$. This game is denoted by $E F_{\kappa}^{c}(A, B)$, and the respective dynamical version by $E F D_{T}^{c}(A, B)$. II wins this game if the end result is a partial chain isomorphism between $A$ and $B$. Obviously, $A \cong \cong_{\text {chain }} B$ iff $I I$ has a winning strategy in $E F_{\kappa}^{c}(A, B)$. Let $\tau$ be the strategy of $I$ in which he systematically lists elements of $A_{\alpha} \cup B_{\alpha}$ for each $\alpha$, smaller values of $\alpha$ before the larger values. Similarly 
to the way $S_{1}$ was defined, let $S_{2}$ be the tree of plays of II against $\tau$ where she has not lost yet. Also, let $K_{2}$ be again the tree of winning strategies of $I I$ in shorter versions of $E F_{\kappa}^{c}(A, B)$ where we limit the length of the game to be an ordinal $<\kappa$. It is again easy to see, as in Lemma 1.1, that $I$ wins $E F D_{\sigma S_{2}}^{c}(A, B), I I$ wins the game $E F D_{K_{2}}^{c}(A, B)$, and $K_{2} \leq S_{2}$. The trees $S_{2}$ and $K_{2}$ do not have $\kappa$-branches, if we assume $A \varlimsup_{\text {chain }} B$. The difference to the previous game is that now, if we assume that $\kappa$ is a strong limit, $\left|\sigma S_{2}\right| \leq \kappa$ and $\left|K_{2}\right| \leq \kappa$, so these are $\kappa$-Trees. Such trees are the topic of Sections 2-6.

The $E F$ game for models $A$ and $B$ of size $\kappa$, needed to prove $L_{\infty \kappa}$-elementary equivalence in the classical sense is the following: players play sequences of elements of $A \cup B$ for $\omega$ steps. The sequences have to be shorter than $\kappa$. This game is denoted by $E F_{\omega}^{<\kappa}(A, B)$, and the respective dynamic version by $E F D_{T}^{<\kappa}(A, B)$. II wins this game if the end result is a partial isomorphism between $A$ and $B$. Player $I I$ has a winning strategy in $E F_{\omega}^{<\kappa}(A, B)$ if and only if the models $A$ and $B$ satisfy (in the classical sense) the same $L_{\infty \kappa}$-sentences, in symbols $A \equiv_{\infty \kappa} B$. Note that $I I$ having a winning strategy in $E F_{\omega}^{<\kappa}(A, B)$ is not a guarantee for the isomorphism of $A$ and $B$ (see [NaSt]). In this respect the games $E F_{\kappa}(A, B)$ and $E F_{\kappa}^{c}(A, B)$ are "better". Assuming $A \not \equiv \neq_{\infty} B$, there is a minimal $\alpha<\left(\kappa^{<\kappa}\right)^{+}$such that $I$ wins $\operatorname{EF}_{\sigma T}^{<\kappa}(A, B)$, where $T$ is the tree of descending sequences of elements of $\alpha$. By determinacy then $I I$ wins $E F D_{T}^{<\kappa}(A, B)$. In this case $|T| \leq \kappa^{<\kappa}$, so $T$ is a $\left(\kappa^{<\kappa}, \omega\right)$-Tree.

Finally, the EF game for chain models $A$ and $B$ of size $\kappa$ and length $\mu$, needed to prove their $L_{\infty \kappa}$-elementary equivalence as chain models (in the sense of $(1)$ ) is the following: players play sequences of length $<\kappa$ of elements of $A$ or $B$ for $\mu$ steps. The sequences have to be contained in some $A_{\alpha} \cup B_{\alpha}$. This game is denoted by $E F_{\mu}^{c,<\kappa}(A, B)$ and the respective dynamic version by $E F D_{T}^{c,<\kappa}(A, B)$. II wins this game if the end result is a partial chain isomorphism between $A$ and $B$. Player $I I$ has a winning strategy in $E F_{\mu}^{c,<\kappa}(A, B)$ if and only if $A$ and $B$ are chain isomorphic. Moreover, if $\mu=\omega$, then both are equivalent to the models $A$ and $B$ satisfying (in the sense of (1)) the same $L_{\infty \kappa}$-sentences. Note the difference to the game $E F_{\omega}^{<\kappa}(A, B)$ in the case $\mu=\omega$. This difference is the whole point of chain models.

Let $\tau$ be a strategy of $\mathrm{I}$ in $E F D_{\mu}^{c,<\kappa}(A, B)$ in which he lists during his move number $\alpha=\nu+2 n<\mu, \nu=\cup \nu$, all elements of $A_{\nu+n} \backslash \bigcup_{\beta<\nu+n} A_{\beta}$, and during move number $\alpha=\nu+2 n+1<\mu, \nu=\cup \nu$, all elements of $B_{\nu+n} \backslash \bigcup_{\beta<\nu+n} B_{\beta}$. Let $S_{4}$ be again the tree of plays of II against $\tau$ where she has not lost yet. Let $K_{4}$ be again the tree of winning strategies of $I I$ in shorter versions of $E F_{\mu}^{c,<\kappa}(A, B)$ where we limit the length of the game to be an ordinal $<\mu$. It is again easy to see that $I$ wins $E F D_{\sigma S_{4}}^{c,<\kappa}(A, B), I I$ wins the game $E F D_{K_{4}}^{c,<\kappa}(A, B)$, and $K_{4} \leq S_{4}$. The trees $S_{4}$ and $K_{4}$ do not have branches of length $\mu$ if $A \approx_{\text {chain }} B$. If, moreover, $\kappa$ is a strong limit, then $\left|\sigma S_{4}\right| \leq \kappa$ and $\left|K_{4}\right| \leq \kappa$. So these are $\kappa$-Trees, but in fact more: they do not even have branches of length $\mu$. So they are $(\kappa, \mu)$-Trees. Such trees are the topic of Section 7. 
In conclusion, the investigation of Scott watersheds for models of cardinality $\kappa$ has led us to study the order of trees without $\kappa$-branches. In the case of chain models of cardinality $\kappa$ singular strong limit of cofinality $\omega$, the relevant trees are moreover $\kappa$-Trees.

For the rest of the paper, unless stated otherwise, let $\kappa$ be a singular cardinal of cofinality $\omega$ and $T$ a $\kappa$-Tree. We also fix an increasing sequence $\left\langle\kappa_{n}: n<\omega\right\rangle$ of regular cardinals converging to $\kappa$.

\section{Ranks and Games}

In this section we introduce a rank operation and show that it characterizes bounded $\kappa$-Trees.

Definition 2.1 For $t \in T$ let $\rho_{T}(t)=\rho(t)$ be defined by recursion on $\alpha$ as follows: $\rho(t) \geq 0$ for any $t \in T$;

$\rho(t) \geq \alpha$ iff for all $\beta<\alpha$ and $n<\omega$ there is $t^{\prime} \geq t$ such that $h\left(t^{\prime}\right) \geq \kappa_{n}$ and $\rho\left(t^{\prime}\right) \geq \beta$.

Example 2.2 Note that $\rho(t) \geq 1$ iff $t$ has extensions of unbounded height in $\kappa$. Let $F$ be the "fan", namely a tree with a single root with $\omega$ maximal branches where each two only intersect at the root, and where there is one branch of length $\kappa_{n}$ for each $n$. Then $F$ is a $\kappa$-Tree, and one can verify that $\rho(F)=1$.

Some basic properties of the rank function are given by the following

Claim 2.3 1. If $t \leq t^{\prime}$ and $\rho\left(t^{\prime}\right) \geq \alpha$, then $\rho(t) \geq \alpha$.

2. If $\delta$ is a limit ordinal and $\rho(t) \geq \alpha$ for all $\alpha<\delta$, then $\rho(t) \geq \delta$.

3. Suppose that $f: T \rightarrow T^{\prime}$ is a reduction between $\kappa$-Trees, and $\rho_{T}(t) \geq \alpha$. Then $\rho_{T^{\prime}}(f(t)) \geq \alpha$.

4. The truth of ' $\rho(t) \geq \alpha$ ' does not depend on the choice of the sequence $\bar{\kappa}=\left\langle\kappa_{n}: n<\omega\right\rangle$.

Proof. (4) Suppose that $\bar{\kappa}^{\prime}=\left\langle\kappa_{n}^{\prime}: n<\omega\right\rangle$ is another sequence with supremum $\kappa$, and define $\rho^{\prime}$ in the same way as $\rho$ but using $\bar{\kappa}^{\prime}$ in place of $\bar{\kappa}$. We show by induction on $\alpha$ that for any $t \in T, \rho(t) \geq \alpha$ iff $\rho^{\prime}(t) \geq \alpha$.

Suppose that $\rho(t) \geq \alpha$ and let $n<\omega$ and $\beta<\alpha$. Let $m<\omega$ such that $\kappa_{m} \geq \kappa_{n}^{\prime}$. Hence there is $t^{\prime} \geq t$ with $h\left(t^{\prime}\right) \geq \kappa_{m}$ and such that $\rho\left(t^{\prime}\right) \geq \beta$. By the inductive assumption we also have $\rho^{\prime}\left(t^{\prime}\right) \geq \beta$. This shows that $\rho^{\prime}(t) \geq \alpha$, and the other direction is symmetric. $\star_{2.3}$ 
Definition 2.4 For any $t \in T$ if there is $\alpha$ such that $\rho(t) \geq \alpha$ but not $\rho(t) \geq \alpha+1$, we say that $\rho(t)=\alpha$. We let $\rho(T)=\rho\left(t^{*}\right)$. It follows from Claim 2.3(1) that if $t \leq t^{\prime}$ then $\rho(t) \geq \rho\left(t^{\prime}\right)$.

The point of the rank operation is that it characterises bounded trees, in the following sense:

Theorem 2.5 $T$ is bounded iff $\rho(T)<\kappa^{+}$.

Proof. In the forward direction, we use the following

Claim 2.6 Suppose that $t \in T$ and $\rho(t) \geq \kappa^{+}$, while $n<\omega$. Then there is $t^{\prime} \geq t$ such that $\rho\left(t^{\prime}\right) \geq \kappa^{+}$and $h\left(t^{\prime}\right) \geq \kappa_{n}$.

Proof. Suppose otherwise, so for every $t^{\prime} \geq t$ of height $\geq \kappa_{n}$ we have $\rho\left(t^{\prime}\right)<\kappa^{+}$. Therefore $\rho(t) \leq \sup _{t^{\prime} \geq t, h(t) \geq \kappa_{n}} \rho(t)<\kappa^{+}$, which is a contradiction. $\star_{2.6}$

So if $\rho\left(t^{*}\right) \geq \kappa^{+}$, we could use Claim 2.6 to build a branch $t^{*}=t_{0} \leq t_{1} \leq t_{2} \ldots$ such that for each $n$ we have $\rho\left(t_{n+1}\right) \geq \kappa^{+}$and $h\left(t_{n+1}\right) \geq \kappa_{n}$. Any maximal branch containing this branch must be a $\kappa$-branch in $T$, which is a contradiction.

In the other direction, suppose that we had a $\kappa$-branch in $T$ and show by induction on $\alpha \leq \kappa^{+}$that $\rho\left(t^{*}\right) \geq \alpha$. Let $t_{0} \leq t_{1} \leq t_{2} \ldots$ be such that $h\left(t_{n}\right) \geq \kappa_{n^{-}}$such a sequence exists by the assumption. The nontrivial case is that $\alpha=\beta+1$. The inductive hypothesis is that for all $n, \rho\left(t_{n}\right) \geq \beta$. The choice of $t_{n}$ 's shows that in fact for all $n$ we have $\rho\left(t_{n}\right) \geq \alpha$, so $\rho\left(t^{*}\right) \geq \alpha$. $\star_{2.5}$

Corollary 2.7 Suppose that $T, T^{\prime}$ are bounded $\kappa$-Trees and $T \leq T^{\prime}$. Then $\rho(T) \leq \rho\left(T^{\prime}\right)$.

Proof. By Theorem 2.5 and Claim 2.3(3). $\star_{2.7}$

It follows from Lemma 2.5 that in a bounded $\kappa$-Tree there is for every $t$ the first $n$ such that $t$ does not have an extension $t^{\prime} \geq t$ of height $\geq \kappa_{n}$ and satisfying $\rho\left(t^{\prime}\right) \geq \rho(t)$. We denote this $n$ by $n_{T}(t)$ or $n(t)$ if $T$ is clear from the context .

Claim 2.8 Suppose that $T$ is a bounded $\kappa$-Tree and $t \leq_{T} t^{\prime}$ satisfy $\rho(t)=\rho\left(t^{\prime}\right)$. Then $n(t) \geq n\left(t^{\prime}\right)$.

Proof. $t$ does not have an extension $t^{\prime \prime}$ of height $\geq \kappa_{n(t)}$ satisfying $\rho\left(t^{\prime \prime}\right) \geq \rho(t)$, so certainly $t^{\prime}$ does not have such an extension. Therefore $n\left(t^{\prime}\right) \leq n(t) . \star_{2.8}$

We introduce a game that can be used to characterise the rank of bounded trees. 
Definition 2.9 Let $T$ be a $\kappa$-Tree and let $t_{0} \in T$. Game $\Gamma_{T}\left(t_{0}\right)$ is defined as follows. Two players I and II play and I commences by $n_{0}<\omega$ and $\beta_{0}<\kappa^{+}$, II replies by $t \geq t_{0}$ of height at least $\kappa_{n_{0}}$. I chooses $n_{1} \geq n_{0}$ and $\beta_{1}<\beta_{0}$. II replies by $t_{1} \geq t$ of height at least $\kappa_{n_{1}}$ etc. The game continues until either of the players can no longer play according to these rules. The winner is the player who played the last move.

We define $\Gamma_{T}\left(\alpha, t_{0}\right)$ for $\alpha<\kappa^{+}$similarly but we require $\beta_{0}<\alpha$.

Note that the above games are determined, because they only involve finitely many moves.

Claim 2.10 If $T$ is a $\kappa$-Tree with root $t^{*}$ then $\rho(T) \geq \alpha$ iff II has a winning strategy in $\Gamma_{T}(\alpha) \stackrel{\text { def }}{=} \Gamma_{T}\left(\alpha, t^{*}\right)$.

Proof. In the forward direction, let II respond to any $\left(n_{m}, \beta_{m}\right)$ by an extension of $t_{m}$ of height at least $\kappa_{n_{m}}$ and rank at least $\beta_{m^{-}}$which is possible by the definition of the rank.

For the other direction, suppose that $\beta<\alpha$ and $n$ are given and that there is no $t \in T$ with $\rho(t) \geq \beta$ and $h(t) \geq \kappa_{n}$. Let $\beta_{0}$ be the first for which there is such $n$, and let $n_{0}=n_{T}(t)$, i.e. it is the first $n$ with this property for $\beta=\beta_{0}$. Start a play of $\Gamma_{T}(\alpha)$ by letting I move with $\left(n_{0}, \beta_{0}\right)$. Because II has a winning strategy, this strategy must respond with $t \in T$ with $h(t) \geq \kappa_{n_{0}}$. However, it must be that $\rho_{T}(t)<\beta_{0}$, so let $\beta_{1}<\beta_{0}$ be the first for which there is no $t_{1} \geq t$ of rank $\geq \beta_{1}$ and let $n_{1}=n_{T}(t)$. Therefore $n_{1} \geq n_{0}$. Let I play $\left(n_{1}, \beta_{1}\right)$. Since II is playing according to the winning strategy, the play can never stop at a move for II. However, the choice of $\left(n_{k}, \beta_{k}\right)$ guarantees that I can respond to each move of II, therefore obtaining an infinite decreasing sequence $\left\langle\beta_{k}: k<\omega\right\rangle$ of ordinals. Contradiction. Therefore $\rho(T) \geq \alpha . \star_{2.10}$

Corollary 2.11 If $T \leq T^{\prime}$ are $\kappa$-Trees and $\alpha<\kappa^{+}$then II wins $\Gamma_{T^{\prime}}(\alpha) \Longrightarrow$ II wins $\Gamma_{T}(\alpha)$.

Proof. By Corollary 2.7 and Claim 2.10. $\star_{2.11}$

Theorem 2.12 If $T$ is a $\kappa$-Tree with root $t^{*}$ then it has a $\kappa$-branch iff II can win all $\Gamma_{T}\left(\alpha, t^{*}\right)$ for $\alpha<\kappa^{+}$.

Proof. In the forward direction, any $\kappa$-Tree with a $\kappa$-branch satisfies $\rho\left(t^{*}\right) \geq \kappa^{+}$, and therefore the conclusion follows from Claim 2.10.

In the backwards direction, again by Claim 2.10 we conclude $\rho\left(t^{*}\right) \geq \kappa^{+}$and hence $T$ cannot be bounded. $\star_{2.12}$ 


\section{Operations on $\kappa$-Trees}

In this section we introduce various operations on trees which will allow us to study bounded trees of a given rank. A reader interested directly in structure results about the class of $\kappa$-Trees may skip this section and use it as a reference.

Notation 3.1 If $T$ is a tree and $t \in T$ we let $T[\geq t]$ stand for $\{s \in T: s \geq t\}$ with the order induced by $T$.

\section{$3.1 \quad$ Topping}

This operation is used to construct from a given tree a tree of the same rank with a long stem. It will be useful when considering the universality problem within a rank.

Definition 3.2 Suppose that $T$ is a bounded $\kappa$-Tree and $\gamma<\kappa$. Let $T_{\lfloor\gamma\rfloor}$ be the tree obtained by taking a stem of length $\gamma$ and putting a disjoint copy of $T$ on top of it. Formally, $T_{\lfloor\gamma\rfloor}$ is $(\{0\} \times \gamma) \cup(\{1\} \times T)$ ordered lexicographically.

Definition $3.3 \mathrm{~A} \kappa$-Tree $T$ with root $t^{*}$ is said to be unfoldable if for no $t \in T \backslash\left\{t^{*}\right\}$ do we have $T \leq T[\geq t]$.

Claim 3.4 Suppose that $T$ is a bounded $\kappa$-Tree and $\beta \leq \gamma<\kappa$.

1. $T_{\lfloor\beta\rfloor} \leq T_{\lfloor\gamma\rfloor}$.

2. If $T$ is unfoldable then $T_{\lfloor\gamma\rfloor} \nless T_{\lfloor\beta\rfloor}$.

3. $\rho\left(T_{\lfloor\gamma\rfloor}\right)=\rho(T)$.

Proof. (1) The identity function is a reduction from $T_{\lfloor\beta\rfloor}$ to $T_{\lfloor\gamma\rfloor}$.

(2) Suppose $f$ is a reduction from $T_{\lfloor\gamma\rfloor}$ to $T_{\lfloor\beta\rfloor}$. Then $f\left(\left(1, t^{*}\right)\right)=(1, t)$ for some $t \neq t^{*}$. Hence $f$ gives rise to a reduction from $T$ to $T[\geq t]$, a contradiction.

(3) Since $T$ is a copy of $T_{\lfloor 0\rfloor}$ we have by $(1)$ that $T \leq T_{\lfloor\gamma\rfloor}$ and therefore $\rho(T) \leq \rho\left(T_{\lfloor\gamma\rfloor}\right)$, by Corollary 2.7 .

Let $\rho(T)=\alpha$. We denote by $t^{*}$ the root of $T$ and by $t^{* *}$ the root of $T_{\lfloor\gamma\rfloor}$. Clearly, $\rho_{T_{\lfloor\gamma\rfloor}}\left(1, t^{*}\right)=\alpha$. By the definition of the rank there is $n>0$ such that $t^{*}$ does not have an extension in $T$ of rank $\geq \alpha$ and height $\geq \kappa_{n}$. Note that for every $(1, s) \in T_{\lfloor\gamma\rfloor}$ we have $\rho_{T\lfloor\gamma\rfloor}((1, s))=\rho_{T}(s)$. Let $m$ be the first such that $\gamma+\kappa_{n-1}<\kappa_{m}$. Suppose that $x \in T_{\lfloor\gamma\rfloor}$ is an extension of $t^{*, *}$ of rank $\geq \alpha$ and height $\leq \kappa_{m}$. Then $x=(1, s)$ for some $s \in T$, Therefore $\rho_{T}(s) \geq \alpha$ and by the choice of $m$, also $h_{T}(s) \geq \kappa_{n}$, a contradiction. In conclusion, $\rho\left(T_{\lfloor\gamma\rfloor}\right) \leq \rho(T)$. $\star_{3.4}$ 


\subsection{Bunching}

The idea of the bunching operation is to produce bounded $\kappa$-Trees of arbitrarily large rank $<\kappa^{+}$. Let $F$ be the fan tree described above.

Definition 3.5 Suppose that $\alpha^{*}<\kappa^{+}$and $\bar{T}=\left\langle T_{i}: i<i^{*}\right\rangle$ are bounded $\kappa$-Trees. Then the bunch $\operatorname{Bn}(\bar{T})$ is the $\kappa$-Tree obtained by putting at the end of every maximal branch of $F$ a node followed by a disjoint family of copies of $T_{i} s$.

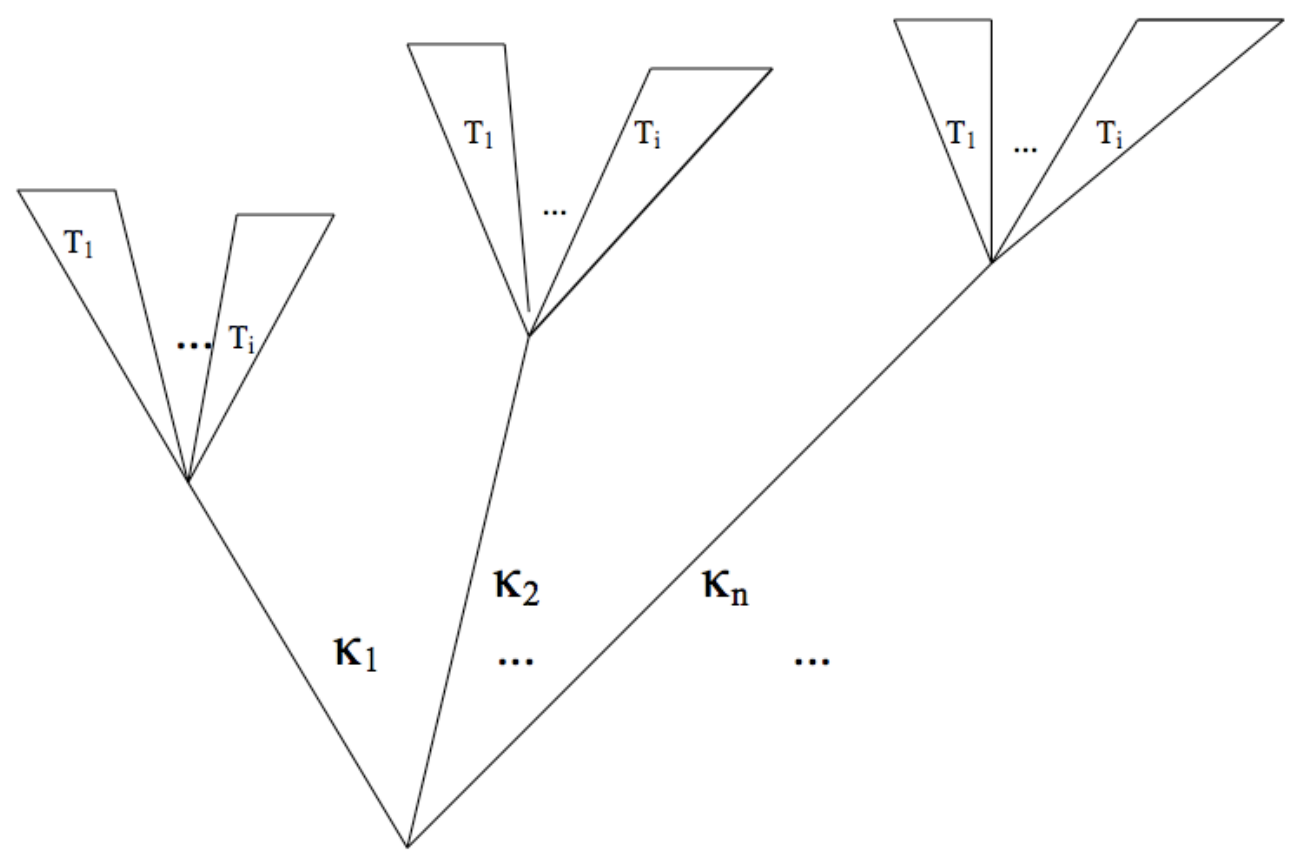

Figure 1: A Bunch $B n\left(\left\langle T_{i}: i<i^{*}\right\rangle\right)$

Claim 3.6 (1) Suppose that $\bar{T}=\left\langle T_{i}: i<i^{*}\right\rangle$ are bounded $\kappa$-Trees and let $T^{*}=\operatorname{Bn}(\bar{T})$. Then $T^{*}$ is bounded and $\rho\left(T^{*}\right) \geq \sup \left\{i<i^{*}: \rho\left(T_{i}\right)+1\right\}$.

(2) If all $T_{i}$ are the same then $\rho\left(T^{*}\right)=\sup \left\{i<i^{*}: \rho\left(T_{i}\right)+1\right\}$

Proof. (1) Clearly, $T^{*}$ is bounded. Let $t^{*}$ be the root of $T^{*}$. First suppose that there is $j$ such that

$$
\alpha=\rho\left(T_{j}\right)=\max \left\{\rho\left(T_{i}\right): i<i^{*}\right\} .
$$

Let $n<\omega$ and let $t$ be the root of the copy of $T_{j}$ which is on top of the branch of $F$ whose length is $\kappa_{n}$. Therefore $\rho_{T^{*}}(t) \geq \alpha$, as follows from Claim 2.3(3) by taking the 
reduction from $T_{j}$ into $T^{*}$ given by mapping $T_{j}$ identically into this particular copy of itself. Therefore $\rho_{T^{*}}\left(t^{*}\right) \geq \alpha+1$.

If there is no such $j$ then we note $\sup \left\{i<i^{*}: \rho\left(T_{i}\right)+1\right\}=\sup \left\{i<i^{*}: \rho\left(T_{i}\right)\right\}$ and repeat the above argument with an arbitrary $\beta<\alpha$ in the place of $\alpha$ above.

(2) Let $T_{i}=T$ for all $i$. Note that then we are in the situation of the first case of the proof of (1), so we can simply follow that argument. $\star_{3.6}$

Corollary 3.7 There is a family $\left\{T_{\alpha}: \alpha<\kappa^{+}\right\}$of bounded $\kappa$-Trees with

$$
\sup \left\{\rho\left(T_{\alpha}\right): \alpha<\kappa^{+}\right\}=\kappa^{+} .
$$

Proof. By induction on $\alpha$ we construct a bounded tree $T_{\alpha}$ satisfying $\rho\left(T_{\alpha}\right) \geq \alpha$. For $\alpha=1$ let $T_{1}=F$. For $\alpha+1$ let $\bar{T}$ consist of a unique element $T_{\alpha}$, and then let $T_{\alpha+1}=\operatorname{Bn}(\bar{T})$. For $\alpha$ limit let $T_{\alpha}=\operatorname{Bn}\left(\left\langle T_{\beta}: \beta<\alpha\right\rangle\right)$. $\star_{3.7}$

In fact, Corollary 3.7 can be improved to obtain a better control on the trees obtained as well as on the ranks. We use the notion of unfoldable trees from Definition 3.3. We isolate a specific property of the trees obtained by the bunching operation which will interest us.

Claim $3.8 \quad$ 1. F is unfoldable.

2. Suppose that $\bar{T}=\left\langle T_{i}: i<i^{*}\right\rangle$ is a sequence of unfoldable $\kappa$-Trees. Then $\operatorname{Bn}(\bar{T})$ is unfoldable.

Proof. (1) has a proof that can easily be extracted from the proof of (2), so let us prove $T$. Let $T^{*}=\operatorname{Bn}(\bar{T})$ and let $t^{*}$ be the root of $T^{*}$. Suppose that $T^{*}$ is not unfoldable, so let $t \neq t^{*}$ be such that $T^{*} \leq T^{*}[\geq t]$ and let $f$ be a reduction witnessing this.

First suppose that $t$ is one of the nodes of the bottom copy of $F$ or the nodes added as an immediate successor of such a branch. Therefore it is a node on a branch of length $\kappa_{n}+1$ for some $n$. Since $T^{*} \leq T^{*}[\geq t]$, in particular the image of the branch $B_{n+1}$ of $F$ of length $\kappa_{n+1}$ is contained in $T^{*}[\geq t]$, and it has to be a branch of length at least $\kappa_{n+1}$. Therefore an end segment of this branch is completely within a copy of exactly one $T_{i}$. Let $s$ be any element of that end segment which is not the root of $T_{i}$. Then in particular we have that $f$ maps the copy of $T_{i}$ on top of $B_{n+1}$ into $T^{*}[\geq s]$, which is isomorphic to $T_{i}\left[\geq s^{\prime}\right]$ for some $s^{\prime}$ not the root of $T_{i}$. This is a contradiction.

Therefore suppose that $t$ is in a copy of some $T_{i}$ on top of some branch of length $\kappa_{n}+1$. Then argue as above with $t$ in place of $s$ to obtain a contradiction. $\star_{3.8}$

Theorem 3.11 below is an improvement of Corollary 3.7. Its proof will feature two variants on the bunching operation which we isolate in the following definition. In fact, 
the operation of a disjoint bunch is sufficient for the purposes of Theorem 3.11, but we describe the mixed bunch as well, as it seems like an interesting variant.

Definition 3.9 (1) Suppose that $\bar{T}=\left\langle T_{n}: n<\omega\right\rangle$ are $\kappa$-Trees. Then the mixed bunch of $\bar{T}$ is the tree $\operatorname{MBn}(\bar{T})$ obtained by putting a copy of $T_{n}$ on top of the branch of length $\kappa_{n}$ of $F$.

(2) Suppose that $\bar{T}=\left\langle T_{i}: i<i^{*}\right\rangle$ are $\kappa$-Trees. Then the disjoint bunch of of $\bar{T}$ is the tree $\operatorname{DBn}(\bar{T})$ obtained by putting a copy of each $T_{i}$ on top of a common root.

Claim 3.10 (1) Suppose that $\bar{T}=\left\langle T_{n}: n<\omega\right\rangle$ are unfoldable $\kappa$-Trees with strictly increasing ranks. Then $T^{*}=\operatorname{MBn}(\bar{T})$ is unfoldable and satisfies $\rho\left(T^{*}\right)=\sup _{n<\omega} \rho\left(T_{n}\right)$. (2) Suppose that $\bar{T}=\left\langle T_{i}: i<i^{*}\right\rangle$ are unfoldable $\kappa$-Trees of increasing rank and $i^{*}<\kappa^{+}$. Then $T^{*}=\operatorname{DBn}(\bar{T})$ is an unfoldable $\kappa$-Tree and satisfies $\rho\left(T^{*}\right)=\sup _{i<i^{*}} \rho\left(T_{i}\right)$.

Proof. The argument is similar to the ones in the proof of Claim 3.6 and Claim 3.8. (1) Suppose that $t \in T^{*} \backslash\left\{t^{*}\right\}$ is such that $T \leq T[\geq t]$. Let $n$ be such that $t$ is on the branch of $F$ of length $\kappa_{n}$ or in the tree $T_{n}$ above that branch. Then the reduction witnessing $T \leq T[\geq t]$ in particular gives rise to a reduction from $T_{n+1}$ into $T_{n\left\lfloor\kappa_{n}\right\rfloor}$, which is a contradiction because $\rho\left(T_{n\left\lfloor\kappa_{n}\right\rfloor}\right)=\rho\left(T_{n}\right)<\rho\left(T_{n+1}\right)$. Therefore $T^{*}$ is unfoldable.

Clearly $T_{n} \leq T^{*}$ for all $n$, and hence $\rho\left(T^{*}\right) \geq \alpha \stackrel{\text { def }}{=} \sup _{n<\omega} \rho\left(T_{n}\right)$. Suppose $\rho\left(T^{*}\right) \geq \alpha+1$ and let $t \neq t^{*}$ be such that $\rho(t) \geq \alpha$. Let $n$ be such that $t$ is on the branch of $F$ of length $\kappa_{n}$ or in the tree $T_{n}$ above that branch. Let $S_{n}$ denote the branch and the tree $T_{n}$ on top of it, so $S_{n}$ is isomorphic to $T_{n\left\lfloor\kappa_{n}\right\rfloor}$ whose rank is $<\alpha$, a contradiction.

(2) Because $i^{*}<\kappa^{+}$we have $\left|T^{*}\right| \leq \kappa$. Suppose that $t \in T^{*} \backslash\left\{t^{*}\right\}$ is such that $T \leq T[\geq t]$ and let $i$ be such that $t$ is in the copy of $T_{i}$ used to construct $T^{*}$. The reduction witnessing $T \leq T[\geq t]$ in particular gives rise to a reduction from $T_{i+1}$ into $T_{i}$, a contradiction.

As in (1), since $T_{i} \leq T^{*}$ for all $i$ we have $\rho\left(T^{*}\right) \geq \alpha \stackrel{\text { def }}{=} \sup _{i<i^{*}} \rho\left(T_{i}\right)$. If $\rho\left(T^{*}\right) \geq \alpha+1$ then there is $t \in T^{*}$ such that $\rho(t) \geq \alpha$ and $t$ is not the root of $T^{*}$. Therefore $t$ is in a copy of some $T_{i}$ and we obtain a contradiction as in (1). $\star_{3.10}$

Theorem 3.11 For every rank $1 \leq \alpha<\kappa^{+}$there is an unfoldable $\kappa$-Tree $T^{\alpha}$ of rank $\alpha$.

Proof. The proof is by induction on $\alpha$. For $\alpha=1$ let $T^{1}=F$. For $\alpha+1$ let $\bar{T}=\left\langle T^{\alpha}\right\rangle$ and let $T^{\alpha+1}=\operatorname{Bn}(\bar{T})$, so $T^{\alpha+1}$ is unfoldable by Claim 3.8(2) and has rank $\alpha+1$ by Claim 3.6(2). For $\alpha$ limit we use Claim 3.10(2) and the induction hypothesis. $\star_{3.11}$ 


\subsection{Cutting branches}

The idea of the following operation is to associate to every $\kappa$-Tree a bounded tree of height $\kappa$ (but possibly of size $\kappa^{+}$).

Definition 3.12 (1) Let $T$ be a $\kappa$-Tree. Let

$$
R(T)=\left\{r^{*}(T)\right\} \cup\left\{(\alpha, t, n): \alpha<\kappa^{+}, t \in T, h(t)<\kappa_{n}\right\},
$$

ordered by letting $(\alpha, t, n)<\left(\alpha^{\prime}, t^{\prime}, n^{\prime}\right)$ if $\alpha>\alpha^{\prime}$ or $\alpha^{\prime}=\alpha, t<t^{\prime}$ and $n \geq n^{\prime}$, and letting every $(\alpha, t, n) \geq r^{*}(T)$.

(2) For $T$ as above and $\alpha^{*}<\kappa^{+}$let $R_{\alpha^{*}}(T)=\left\{r^{*}(T)\right\} \cup\left\{(\alpha, t, n) \in R(T): \alpha \leq \alpha^{*}\right\}$, with the order inherited from $R(T)$.

Lemma 3.13 Let $T$ be a $\kappa$-Tree.

1. $R(T)$ is a tree of height at most $\kappa$ with no $\kappa$ branches, and so is every $R_{\alpha^{*}}(T)$.

2. $T$ is bounded iff $T \leq R(T)$. If $T$ is bounded then $T \leq R_{\rho(T)}(T)$.

Proof. (1) If $(\alpha, t, n) \in R(T)$ then the predecessors of $\left(\alpha^{\prime}, t^{\prime}, n^{\prime}\right)$ are clearly well ordered in $R(T)$. Suppose that $\left\langle\left(\alpha_{i}, t_{i}, n_{i}\right): i<\kappa\right\rangle$ is increasing in $R(T)$. Then there must be $i^{*}<\kappa$ such that all $\alpha_{i}$ for $i \geq i^{*}$ are a fixed $\alpha$. Therefore $\left\langle t_{i}: i^{*} \leq i<\kappa\right\rangle$ is increasing in $T$, and all $t_{i}$ have height $<\kappa_{n}$, a contradiction. We argue similarly for $R_{\alpha^{*}}(T)$.

(2) In the forward direction, let $f(t)=\left(\rho_{T}(t), t, n_{T}(t)\right)$. This is well defined because $T$ is bounded, as by Theorem 2.5. If $t<t^{\prime}$ we have $\rho(t) \geq \rho\left(t^{\prime}\right)$ so if $\rho(t)>\rho\left(t^{\prime}\right)$ then $f(t)<f\left(t^{\prime}\right)$ and if $\rho(t)=\rho\left(t^{\prime}\right)$ then $t<t^{\prime}$ and $n(t) \geq n\left(t^{\prime}\right)$ (by Claim 2.8). Therefore $f$ is a reduction. In the other direction, since $R(T)$ has no branches of size $\kappa$ and $T \leq R(T)$, $T$ cannot have any branches of size $\kappa$.

The last sentence follows from the proof of the forward direction above. $\star_{3.13}$

\section{Twins and other cofinalities}

We consider two functors which make a connection between $\kappa$-Trees and trees of size $\kappa$ and height $\omega$. It is convenient to assume that $\kappa_{0}=0$.

Definition 4.1 Suppose that $T$ is a $\kappa$-Tree. Then the small twin $\operatorname{tw}(T)$ of $T$ is the tree $T^{\prime}$ of height $\omega$ such that $\operatorname{lev}_{n}\left(T^{\prime}\right)=\operatorname{lev}_{\kappa_{n}}\left(T^{\prime}\right)$ with the order induced from $T$. If $T$ is a tree of size $\kappa$ and height $\omega$ then the big twin $\operatorname{Tw}(T)$ is a normal $\kappa$-Tree $T$ such that $\operatorname{lev}_{\kappa_{n}}(T)=\operatorname{lev}_{n}\left(T^{\prime}\right)$ and for every $n$ and $t \in T^{\prime}$ of height $n$, the set of immediate successors of $t$ is in a bijective correspondence $f_{t}$ with $\left\{t^{\prime} \in T: h\left(t^{\prime}\right)=n+1 \& t \leq t^{\prime}\right\}$. 
For any $\alpha \in\left[\kappa_{n}+1, \kappa_{n+1}\right)$, every point of height $\alpha$ in $T^{\prime}$ has exactly one immediate successor, and finally we have that for $t$ of height $\kappa_{n}$ and $t^{\prime}$ of height $\kappa_{n+1}, t \leq_{T}^{\prime} t^{\prime}$ iff $t \leq_{T} t^{\prime}$. We also have that $s \leq f_{t}(s)$ whenever $s$ is an immediate successor of $t$.

Basic properties of the twinning functors are the following:

Claim 4.2 1. For any $\kappa$-Tree $T$ we have $\operatorname{tw}(T)$ has an infinite branch iff $T$ is unbounded. If $T^{\prime}$ is a tree of height $\omega$ and size $\kappa$, then $\mathrm{Tw}\left(T^{\prime}\right)$ is unbounded iff $T$ has an infinite branch.

2. $\operatorname{tw}(\mathrm{Tw})(T)=T$, for any relevant $T$.

3. If $T \leq T^{\prime}$ are $\kappa$-Trees then $T \leq T^{\prime} \Longrightarrow \operatorname{tw}(T) \leq \operatorname{tw}\left(T^{\prime}\right)$, by the same witnessing function.

4. Suppose that $T \leq T^{\prime}$ are trees of height $\omega$ and size $\kappa$. Then $\operatorname{Tw}(T) \leq \operatorname{Tw}\left(T^{\prime}\right)$.

Proof. (4) Let $f: T \rightarrow T^{\prime}$ be a reduction. The Twinning operation gives an embedding of $T$ into $\operatorname{Tw}(T)$ which maps level $n$ of $T$ into the level $\kappa_{n}$ of $\operatorname{Tw}(T)$, and similarly for $T^{\prime}$. Let us denote both of these mappings as $g$. Now we define $f^{*}: \operatorname{Tw}(T) \rightarrow \operatorname{Tw}\left(T^{\prime}\right)$ so that $f^{*}(g(t))=g(f(t))$ for all $t \in T$. Notice that $f^{*}$ is a partial reduction and if $h_{\operatorname{Tw}(T)}(t)=\kappa_{n}$ then $h_{\operatorname{Tw}\left(T^{\prime}\right)}\left(f^{*}\right)(t)=\kappa_{m}$ for some $m \geq n$. Also note that $\operatorname{Tw}(T)$ cannot have a maximal branch that terminates at a level $\alpha \notin\left\{\kappa_{n}: n<\omega\right\}$. Therefore we can extend $f^{*}$ to a full reduction from $\operatorname{Tw}(T)$ to $\operatorname{Tw}\left(T^{\prime}\right)$. $\star_{4.2}$

\section{Universals}

The universality number of a class of trees is the smallest cardinal number $\theta$ such that there is a family $\mathcal{U}$ of $\theta$ many members of the class, satisfying that for every $T$ in the class there is $T^{\prime} \in \mathcal{U}$ such that $T \leq T^{\prime}$. A family $\mathcal{U}$ satisfying the latter property is called a universal family.

We can obtain some results about universality of $\kappa$-Trees as a consequence of the observations made in the previous section.

Claim 5.1 The universality number of the class of all $\kappa$-Trees is larger or equal to the universality number of the class of all trees of size $\kappa$ and height $\omega$. The same is true if we restrict the former class to bounded members, and the latter to members with no infinite branch. 
Proof. Suppose that $\mathcal{U}$ is a universal family for the class of all $\kappa$-Trees. Let

$$
\mathcal{U}^{\prime}=\{\operatorname{tw}(T): T \in U\} .
$$

Suppose that $T$ is any tree of size $\kappa$ and height $\omega$. Therefore there is $T^{*} \in \mathcal{U}$ such that $\operatorname{Tw}(T) \leq T^{*}$. Hence $T=\operatorname{Tw}(\operatorname{tw}(T)) \leq \mathrm{twT}^{*}$, by Claim 4.2(2) and (3) above. Therefore $\mathcal{U}^{\prime}$ is a universal family for the class of all trees of size $\kappa$ and height $\omega$. The latter statement is proved in the same way. $\star_{5.1}$

The following gives an idea about the universality number of $\kappa$-Trees and bounded $\kappa$-Trees.

Claim 5.2 (1) The universality number of the class of all $\kappa$-Trees is 1.

(2) The universality number of the class of all bounded $\kappa$-Trees is at least $\kappa^{+}$.

Proof. (1) Let $T$ be any $\kappa$-Trees and view $\kappa$ as a $\kappa$-Tree with one unique branch. We define $f: T \rightarrow \kappa$ by letting $f(t)=\alpha$ if $h(t)=\alpha$. This is clearly a reduction.

(2) Suppose this universality number is 1 , as demonstrated by a $T^{*}$. Let $\alpha=\rho(T)$. The bunching operation $\bar{T}=\left\langle T^{*}\right\rangle$ gives rise to a bounded $\kappa$-Tree with rank $T$ at least $\alpha+1$. However $T \leq T^{*}$, in contradiction with Corollary 2.7.

Suppose now that the universality number is some $\lambda \in[2, \kappa]$, as exemplified by trees $\left\{T_{i}: i<\lambda\right\}$. Then we can construct a bounded $\kappa$-Tree $T^{*}$ by defining a root and $\lambda$ many successors of it, on top of $i$-th of which we add a copy of $T_{i}$. This is a $\kappa$-Tree which reduces all $T_{i}$. Therefore the universality number is not in $[2, \kappa] . \star_{5.2}$

We now have

Theorem 5.3 The universality number of the class of all bounded $\kappa$-Trees is exactly $\kappa^{+}$.

Proof. We exhibit a universal family of size $\kappa^{+}$. This suffices by Claim 5.2(2). We consider the ordinal $\kappa$ as a $\kappa$-Tree. Because this tree has a branch of length $\kappa$, every bounded $\kappa$-Tree $T \leq$-embeds into it, simply by mapping every element of $T$ into its height. Let now $T_{\alpha}=R_{\alpha}(\kappa)$, for $\alpha<\kappa^{+}$(recall the branch cutting operation from Subsection 3.3). Therefore $\left\{T_{\alpha}: \alpha<\kappa^{+}\right\}$is a family of bounded $\kappa$-Trees, and we now claim that it is universal.

Let $T$ be any bounded $\kappa$-Tree and let $f: T \rightarrow \kappa$ be a reduction. Let $\alpha=\rho(T)+1$, therefore $\alpha<\kappa^{+}$. Define $g: T \rightarrow T_{\alpha}$ by letting $g(t)=\left(\rho_{T}(t), f(t), n_{T}(t)\right)$. If $t<t^{\prime}$ in $T$ then either $\rho(t)>\rho(t)^{\prime}$, or $\rho(t)=\rho(t)^{\prime}$ and $f(t)<f^{\prime}(t)$. At any rate, $g(t)<g\left(t^{\prime}\right)$. $\star_{5.3}$

Given the above definite results above universality within the class of bounded $\kappa$ Trees we may wonder if there are universality results about the bounded $\kappa$-Trees within the same rank. We have the following: 
Theorem 5.4 (1) For every $1 \leq \alpha<\kappa^{+}$the universality number of the class of $\kappa$-Trees of rank $\alpha$ is exactly $\omega$. Moreover, for every such $\alpha$ there is a single unfoldable $\kappa$-Tree $T^{\alpha}$ of rank $\alpha$ such that $\left\{T_{n}^{\alpha} \stackrel{\text { def }}{=} T_{\left\lfloor\kappa_{n}\right\rfloor}^{\alpha}\right\}$ is a minimal universal family for $\kappa$-Trees of rank $\alpha$.

(2) For every $1 \leq \alpha<\kappa^{+}$there is a single unfoldable $\kappa$-Tree $T_{\alpha}^{*}$ of rank $\alpha+1$ which is universal for $\kappa$-Trees of rank $\alpha$.

Proof. We commence with a simple lemma.

Lemma 5.5 Suppose that $\alpha<\kappa^{+}$and that $\mathcal{F}$ is a family of $\kappa$-Trees of rank $\alpha$ universal for $\kappa$-Trees of rank $\alpha$. Then for every $\beta<\alpha$ and $\kappa$-Tree $T$ of rank $\beta, T$ embeds into some member of $\mathcal{F}$.

Proof. For such $\beta, T$ let $T^{*}$ be the disjoint bunch of $T$ and any tree of rank $\alpha$. Clearly $T^{*}$ has rank $\alpha$, and therefore it embeds into some member of $\mathcal{F}$. The restriction of the witnessing reduction to $T$ demonstrates the lemma. $\star_{5.5}$

We now prove the theorem by induction on $\alpha$, proving (1) and (2) simultaneously.

(1) We distinguish several cases of the induction.

For $\alpha=1$ let $T^{1}=F$, and so $T_{n}^{1}=F_{\left\lfloor\kappa_{n}\right\rfloor}$. By Claim 3.8(1) trees $F$ is unfoldable and by Claim 3.4(3) $T_{n}^{1}$ are of rank 1. By Claim 3.4(2) we have $T_{n+1} \not \leq T_{n}$, so there is no proper subset of $\left\{T_{n}^{1}: n<\omega\right\}$ which can be universal. We now have to prove that every $\kappa$-Tree $T$ of rank 1 embeds into some $T_{n}$. Let $T$ be such a tree.

By the definition of the rank, there is $n=n_{T}$ such that for every $t \in T$ of height $\geq n$ we have $\rho(t)<1$. We define $f: T \rightarrow T_{n}^{1}$ by letting for $s \in T$, if $h(s)<\kappa_{n}, f(s)$ be the node on the stem of $T_{n}^{1}$ of height $h(s)$. The choice of $n$ implies that for every $t \in T$ with $h(t) \geq n$ we have that the whole tree $T[\geq t]$ has height $<\kappa$. Therefore for every such $t$ we can choose $m$ with $h(t)<\kappa_{m}$ and define $f$ on $T[\geq t]$ to carry all points $s$ to the branch of $F$ of length $\kappa_{m}$, assigning to each $s$ the unique point $f(s)$ such that $h_{F}(f(s))=h_{T[\geq t]}(s)$.

Let $1<\alpha<\kappa^{+}$.

Case 1. $\alpha=\beta+1$ for some $\beta$. We claim that letting $T^{\alpha}=T_{\beta}^{*}$ we obtain the desired conclusion. By (2) at $\beta$ we have that $T^{\alpha}$ is unfoldable and has rank $\alpha+1$. Therefore by Claim 3.4(2) we have $T_{n}^{\alpha} \not \leq T_{n+1}^{\alpha}$. Like in the case $\alpha=1$, for a given tree $T$ of rank $\alpha$ we let $n=n_{T}$ and define a reduction $f: T \rightarrow T_{\alpha\left\lfloor\kappa_{n}\right\rfloor}^{*}$ by mapping all $s$ of height $<\kappa_{n}$ to the stem. On the other hand, for any $t \in T$ of height $\geq \kappa_{n}$ we have that the rank of $T[\geq t]$ is at most $\beta$, and so by Lemma 5.5, $T[\geq t]$ embeds into $T_{\alpha}^{*}$.

Case 2. $\alpha$ is a limit ordinal. Let $T^{\alpha}=\operatorname{DBn}\left(\left\langle T^{\beta}: \beta<\alpha\right\rangle\right)$, so by Claim 3.10(2), $T^{\alpha}$ is unfoldable and has rank $\alpha$. The rest of the proof follows closely the proof of Case 1 .

(2) If we are given $T^{\alpha}$ let $T_{\alpha}^{*}$ be $\operatorname{DBn}\left(\left\langle T_{n}^{\alpha}: n<\omega\right\rangle\right)$. It is easily seen that $T_{\alpha}^{*}$ has rank $\alpha+1$, it is unfoldable and satisfies $T_{n}^{\alpha} \leq T_{\alpha}^{*}$ for all $n$. Therefore it is universal for trees of rank $\alpha . \star_{5.4}$ 


\section{Incomparable $\kappa$-Trees}

In this section we are concerned with the antichains of $\kappa$-Trees. We commence by showing that consistently there can be large antichains of $\kappa$-Trees in every rank $\geq 2$.

We shall use the following useful and well known claim.

Claim 6.1 Suppose that $T$ and $T^{\prime}$ and $T \leq T^{\prime}$. Then there is a reduction $f: T \rightarrow T^{\prime}$ satisfying $h(t)=h(f(t))$ for all $t \in T$.

Proof. Let $g: T \rightarrow T^{\prime}$ be any reduction and define $f(t)=g \uparrow h(t)$. Note that the fact that $g$ is a reduction implies that $h(g(t)) \geq g(t)$ for any $t \in T$, so $f$ is well defined. If $s<_{T} t$ then $g(s)<_{T^{\prime}} g(t)$, and hence $g(s) \uparrow h(s)=g(t) \uparrow h(s)<g(t)$, but since $h(s)<h(t)$ we also obtain $g(s) \uparrow h(s)<g(t) \uparrow h(s)$. Therefore $f$ is a reduction. $\star_{6.1}$

We call the reductions satisfying the requirement of Claim 6.1 level preserving reductions.

Theorem 6.2 Suppose $\lambda, \theta$ are cardinals such that $\mu \stackrel{\text { def }}{=} 2^{\lambda^{<} \theta} \leq \kappa$, and $\rho<\kappa^{+}$. Then there is a family of $\mu$ many pairwise incomparable $\kappa$-Trees of rank $\rho$.

Proof. Let $\left\langle S_{\alpha}: \alpha<\mu\right\rangle$ be a family of copies of the tree ${ }^{\theta>} \lambda$, therefore each $S_{\alpha}$ is a tree of size $\kappa$-Tree (here we have used $\left.\lambda^{<\theta} \leq \kappa\right)$. Let $\left\{f_{i}: i<\mu\right\}$ list all level preserving reductions from $S_{\alpha}$ into $S_{\beta}$ for $\alpha \neq \beta<\mu$. We shall extend each $S_{\alpha}$ into a tree $T_{\alpha}$ by selecting certain cofinal branches of $S_{\alpha}$ and attaching a copy of a $\kappa$-Tree of rank $\rho$ on top of it, as described below. The purpose of these extensions is to assure that no $f_{i}$ extends into a reduction between $T_{\alpha}$ and $T_{\beta}$ for $\alpha \neq \beta$. Since we are using up to $\mu$ cofinal branches, we are again using $\mu \leq \kappa$ to assure that $T_{\alpha}$ 's are $\kappa$-Trees.

Let $\left\{T_{j}^{*}: j<\omega\right\}$ list a universal family of trees of rank $\rho$, which exists by Theorem 5.4. By induction on $i<\mu$ we consider $f_{i}$. The inductive hypothesis is that for each $\alpha<\mu$ and $j<i$ we have a tree $S_{\alpha}^{j}$ such that $S_{\alpha}^{0}=S_{\alpha}$ and $\left\langle S_{\alpha}^{j}: j<i\right\rangle$ forms a sequence of trees that end-extend each other, while in the union $S_{\alpha}^{0, i}$ the tree $S_{\alpha}$ has been extended by putting terminal nodes and a copy of some $\kappa$-Tree of rank $\leq \rho$ on top of $<\mu$ cofinal branches of the original $S_{\alpha}$. We assume that each cofinal branch of $S_{\alpha}$ has been extended at most once.

At stage $i$ of the induction, there are unique $\alpha, \beta$ such that $f_{i}: S_{\alpha} \rightarrow S_{\beta}$. For $\gamma \notin\{\alpha, \beta\}$ let $S_{\gamma}^{i}=S_{\gamma}^{0, i}$. Next we choose a cofinal branch $b_{\alpha}^{i}$ in $S_{\alpha}$ which still does not have a terminal node in $S_{\alpha}^{0, i}$ (this is possible since there are $\mu$ many cofinal branches in $S_{\alpha}$, of which we only used $<\mu$ ). Since $f_{i}$ is a level preserving reduction, the image $b_{\beta}^{i}=f^{\prime} b_{\alpha}^{i}$ is a cofinal branch of $S_{\beta}$.

The first possibility is that $b_{\beta}^{i}$ still does not have a terminal node in $S_{\beta}^{0, i}$. Then extend $S_{\alpha}^{0, i}$ to $S_{\alpha}^{i}$ by putting a terminal node and a copy of $T_{0}^{*}$ on top of $b_{\alpha}^{i}$ if $\rho>1$, or $F_{\lfloor 1\rfloor}$ if 
$\rho=1$. Extend $S_{\beta}^{0, i}$ to $S_{\beta}^{i}$ by putting a terminal node and a copy of the fan $F$ on top of $b_{\beta}^{i}$.

Note that $f_{i}$ cannot be extended to a level preserving reduction from $S_{\alpha}^{i}$ to $S_{\beta}^{i}$ because any such reduction would have to move a $\kappa$-Tree of rank $\rho$ into $F$, or $F_{\lfloor 1\rfloor}$ if $\rho=1$ into $F$, which is in contradiction with Claim 2.3. The second possibility is that there is already a $\kappa$-Tree $T$ of rank $\delta \leq \rho$ on top of $b_{\beta}^{i}$. By the choice of the family $\left\{T_{j}^{*}: j<\omega\right\}$ there must be $j$ such that $T_{j}^{*}$ does not embed into $T$. We choose some such $j$ and place $T_{j}^{*}$ on top of $b_{\alpha}^{i}$.

At the end let $T_{\alpha}$ be the union of all $S_{\alpha}^{i}$. It is clear that $\rho\left(T_{\alpha}\right) \geq \rho$. Since $\kappa$ is of cofinality $\omega$ we cannot have $\mu=\kappa$, by König's Lemma. Therefore $\mu<\kappa$ If there is a reduction from $T_{\alpha}$ to $T_{\beta}$ for some $\alpha \neq \beta$ then there is a level preserving reduction $f$, by Claim 6.1. However, $f$ would have to extend some $f_{i}$, which was made impossible by the step $i$ of the induction. $\star_{6.2}$

\section{$7 \quad$ A covering theorem for $\lambda^{\mathrm{cf}(\lambda)}$}

In this section we prove some results about $(\lambda, \kappa)$-Trees, i.e. trees of singular cardinality $\lambda$ without branches of length $\kappa=\operatorname{cf}(\lambda)$. Such trees arise from consideration of the game $E F_{\kappa}^{c,<\lambda}(A, B)$ on chain models $A$ and $B$ of length $\kappa$. In particular, we prove a covering theorem which can be seen as a generalization of the covering theorem of the Baire space $\omega^{\omega}$.

We fix $\lambda$ strong limit singular of cofinality $\kappa$, along with a continuous cofinal sequence $\left\langle\lambda_{\xi}: \xi<\kappa\right\rangle$ of cardinals in $\lambda$ such that $2^{\lambda_{\xi}} \leq \lambda_{\xi+1}$. We shall throughout make the usual identification between ${ }^{\kappa} \lambda$ and $\lambda^{\kappa}$.

We equip the set $\lambda^{\kappa}$ with the topology generated by the neighbourhoods

$$
N(f, \beta)=\{g: \kappa \rightarrow \lambda: g \uparrow \beta=f \uparrow \beta\}
$$

for $f \in{ }^{\kappa} \lambda$ and $\beta<\lambda$. Note that in this topology there is a dense subset of $\lambda^{\kappa}$ of size $\left|\bigcup_{\xi<\kappa} \lambda^{\lambda_{\xi}}\right|=\lambda$, the topology is 0-dimensional and each open set is the union of $\lambda$ closed sets. We make an expected definition:

Definition 7.1 $A$ set $A \subseteq \lambda^{\kappa}$ is $\Pi_{1}^{1}$ if there is an open set $B \subseteq \lambda^{\kappa} \times \lambda^{\kappa}$ (in the product topology where each copy of $\lambda^{\kappa}$ has the topology described above) such that for every $f \in{ }^{\kappa} \lambda$

$$
f \in A \Longleftrightarrow \forall g((f, g)) \in B) .
$$

A set is $\Sigma_{1}^{1}$ if its complement is $\Pi_{1}^{1}$ and it is $\Delta_{1}^{1}$ if it is both $\Pi_{1}^{1}$ and $\Sigma_{1}^{1}$. 
Now we introduce some notation:

Let $\mathcal{T}$ denote the class of trees of cardinality $\leq \lambda$ and height $\leq \kappa$ with no branches of length $\kappa$. Let pair : $\lambda \times \lambda \rightarrow \lambda$ be the standard bijection. If $f, g \in{ }^{\kappa} \lambda$, let $\langle f, g\rangle \in{ }^{\kappa} \lambda$ be a function such that $\langle f, g\rangle(\operatorname{pair}(\alpha, \beta))=\operatorname{pair}(f(\alpha), g(\beta))$ for $\alpha, \beta \in \lambda$. Let $\sigma:{ }^{\kappa>} \lambda \rightarrow \lambda$ be a bijection.

Now we shall fix a bijection $H$ between ${ }^{\kappa}(\mathcal{P}(\lambda))$ and ${ }^{\kappa} \lambda$ which has the following properties. Namely, identifying $\mathcal{P}(\lambda)$ with ${ }^{1} \mathcal{P}(\lambda)$ the function $H$ induces an injection $F$ from $\mathcal{P}(\lambda)$ to ${ }^{\kappa} \lambda$. We require this injection $F$ to have the property that there is a sequence $\left\langle F_{\xi}: \xi<\kappa\right\rangle$ of injections $F_{\xi}: \mathcal{P}\left(\lambda_{\xi+1} \backslash \lambda_{\xi}\right) \rightarrow \lambda_{\xi+2} \backslash \lambda_{\xi+1}$ such that for any $A \subseteq \lambda$ the value $F(A) \in{ }^{\kappa} \lambda$ is a function such that $F(A)\left(\lambda_{\xi}\right)=F_{\xi}\left(A \cap\left(\lambda_{\xi+1} \backslash \lambda_{\xi}\right)\right)$. Such an $H$ is easy to find, by constructing first the functions $F_{\xi}$. For most of the this sections we shall only work with the function $F$, but the whole of function $H$ will be needed once we start talking about chain models in Theorem 7.8 .

If $f: \kappa \rightarrow \lambda$, we denote the initial segment $\langle f(0), \ldots, f(\beta), \ldots\rangle_{\beta<\alpha}$ by $\bar{f}(\alpha)$. By a tree on $\lambda^{<\kappa}$ we mean a subset of $\lambda^{<\kappa}$ which is closed under initial segments. Respectively, a tree on $\lambda^{<\kappa} \times \lambda^{<\kappa}$ we mean a set $T$ of pairs $(\bar{f}(\alpha), \bar{g}(\alpha))$ such that if $(\bar{f}(\alpha), \bar{g}(\alpha))$ and $\beta<\alpha$, then $(\bar{f}(\beta), \bar{g}(\beta))$.

Example 7.2 Let $\operatorname{Tr}=\left\{F\left(\sigma^{\prime \prime} T\right): T\right.$ is a tree on $\left.\lambda^{<\kappa}\right\}$. Therefore $\operatorname{Tr} \subseteq \lambda^{\kappa}$ and we claim that it is closed. Suppose that $f$ is in the closure of $\operatorname{Tr}$, hence for every $\beta<\lambda$ there is $g_{\beta} \in N(f, \beta)$ and some tree $T_{g \beta}$ such that $F\left(\sigma^{\prime \prime} T_{g \beta}\right)=g_{\beta}$. If $\zeta<\xi_{1}<\xi_{2}$ then $g_{\lambda_{\xi_{1}}}\left(\lambda_{\zeta}\right)=g_{\lambda_{\xi_{1}}}\left(\lambda_{\zeta}\right)=f\left(\lambda_{\zeta}\right)$, therefore $F_{\zeta}\left(\sigma^{\prime} T_{g_{\lambda_{1}}} \cap\left(\lambda_{\zeta+1} \backslash \lambda_{\zeta}\right)\right)=F_{\zeta}\left(\sigma^{*} T_{g_{\xi_{2}}} \cap\left(\lambda_{\zeta+1} \backslash \lambda_{\zeta}\right)\right)$. Let $A=\bigcup_{\zeta<\kappa} F_{\zeta}^{-1}\left(\sigma^{"} T_{g_{\lambda_{\xi}}} \cap\left(\lambda_{\zeta+1} \backslash \lambda_{\zeta}\right)\right)$, where $\xi \in(\zeta, \kappa)$ is arbitrary. The discussion above shows that $A$ is well defined. Let $T^{*}=\sigma^{-1}(A)$, so $T^{*} \subseteq \lambda^{<\kappa}$. Now if $g \in T^{*}$ and $h$ is an initial segment of $g$ we have $\sigma(g) \in A$ and therefore for some $\zeta<\xi<\kappa$ we have $\sigma(g) \in F_{\zeta}^{-1}\left(\sigma^{\prime} T_{g_{\lambda_{\xi}}} \cap\left(\lambda_{\zeta+1} \backslash \lambda_{\zeta}\right)\right)$. Hence $\sigma(g) \in \sigma^{\prime} T_{g_{\lambda_{\xi}}}$ and so $g \in T_{g_{\lambda_{\xi}}}$. Therefore $h \in T_{g_{\lambda_{\xi}}}$ and we can show that $h \in T^{*}$ by reversing the implications. Therefore $T^{*}$ is a tree and by its definition $F\left(T^{*}\right)=f$, showing that $f \in \mathrm{Tr}$.

If $f=F\left(\sigma^{\prime} T\right) \in \operatorname{Tr}$ we denote the tree $T$ by $T_{f}$. Let

$$
\mathcal{T O}=\left\{f: T_{f} \text { has no branches of length } \kappa\right\} .
$$

It can be checked that this set is $\Pi_{1}^{1}$.

Proposition 7.3 (Boundedness Theorem) Suppose $B \subseteq \mathcal{T O}$ is $\Sigma_{1}^{1}$. Then there is $g \in \mathcal{T O}$ such that $T_{f} \leq T_{g}$ for all $f \in B$.

Proof. If $T$ is a tree on $\lambda^{<\kappa} \times \lambda^{<\kappa}$ and $f \in{ }^{\kappa} \lambda$, let

$$
T(f)=\{\bar{g}(\alpha):(\bar{f}(\alpha), \bar{g}(\alpha)) \in T\} .
$$


Note that $|T(f)| \leq \lambda$. Let $S=\left\{(\bar{f}(\alpha), \bar{g}(\alpha)): f \in \operatorname{Tr}, \bar{g}(\alpha) \in T_{f}\right\}$. Now $S(f)=T_{f}$ for all $f \in \operatorname{Tr}$ and

$$
f \in \mathcal{T O} \Longleftrightarrow S(f) \text { has no branches of length } \kappa \text {. }
$$

Let $T$ be a tree such that

$$
f \in B \Longleftrightarrow T(f) \text { has a branch of length } \kappa \text {. }
$$

Let $T^{\prime}$ be the tree of triples $(\bar{f}(\alpha), \bar{g}(\alpha), \bar{h}(\alpha))$ such that $\bar{g}(\alpha) \in S(f)$ and $\bar{h}(\alpha) \in T(f)$. If $T^{\prime}$ has a branch of length $\kappa$ then we get $f \in B \backslash \mathcal{T O}$. To prove $T_{f} \leq T^{\prime}$ for all $f \in B$, let $f \in B$ be arbitrary. Let $(\bar{h}(\alpha))_{\alpha<\kappa}$ be a branch of length $\kappa$ in $T(f)$. For $\bar{g}(\alpha) \in S(f)$ let

$$
\phi(\bar{g}(\alpha))=(\bar{f}(\alpha), \bar{g}(\alpha), \bar{h}(\alpha)) .
$$

Now $\phi: S(f) \rightarrow T^{\prime}$ is order-preserving.

To prove the corresponding result for all $\Pi_{1}^{1}$-sets, we present the basic tree-representation of $\Pi_{1}^{1}$-sets. This emerges in the same natural way as in the space ${ }^{\omega} \omega$.

Lemma 7.4 $A$ set $A \subseteq{ }^{\kappa} \lambda$ is $\Pi_{1}^{1}$ if and only if there is a relation $R \subseteq \lambda^{<\kappa} \times \lambda^{<\kappa}$ such that

$$
f \in A \Longleftrightarrow \forall g \exists \alpha R(\bar{f}(\alpha), \bar{g}(\alpha)) .
$$

Proof. If $A$ and $B$ satisfy (2), then

$$
R=\{(\bar{f}(\alpha), \bar{g}(\alpha)): N((f, g), \alpha) \subseteq B\}
$$

satisfies (3). Conversely, if $A$ and $B$ satisfy (3), then

$$
B=\bigcup\{N((f, g), \alpha): R(\bar{f}(\alpha), \bar{g}(\alpha))\}
$$

satisfies (2).

Proposition 7.5 Let $A \subseteq{ }^{\kappa} \lambda$. A is $\Pi_{1}^{1}$ if and only if there is a tree $T$ on $\lambda^{<\kappa} \times \lambda^{<\kappa}$ such that

$$
f \in A \Longleftrightarrow T(f) \text { has no branches of length } \kappa .
$$

Proof. Suppose $A$ is $\Pi_{1}^{1}$ and $R$ is as in (3). Let

$$
T=\{(\bar{f}(\alpha), \bar{g}(\alpha)): \forall \beta \leq \alpha \neg R(\bar{f}(\alpha), \bar{g}(\alpha))\} .
$$

Hence $f \in A$ if and only if $T(f)$ has no branches of length $\kappa$. For the converse, suppose such a $T$ exists. Let

$$
R(\bar{f}(\alpha), \bar{g}(\alpha)) \Longleftrightarrow \exists \beta \leq \alpha(\bar{f}(\beta), \bar{g}(\beta)) \notin T .
$$


Now $f \in A$ if and only if $\forall g \exists \alpha R(\bar{f}(\alpha), \bar{g}(\alpha))$. Thus $A$ is $\Pi_{1}^{1}$.

Suppose $A$ is $\Pi_{1}^{1}$ and $T$ is a tree such that (4) holds. If $g \in \mathcal{T O}$, let

$$
A^{T, g}=\left\{f \in{ }^{\kappa} \lambda: T(f) \leq T_{g}\right\} .
$$

Then clearly, $A^{T, g} \subseteq A$ for $g \in \mathcal{T O}$, and $\lambda^{<\kappa}=\lambda$ implies $\bigcup_{g \in \mathcal{T} \mathcal{O}} A^{T, g}=A$. Using the functions $\sigma$ and $F$ it is not hard to see that the sets $A^{T, g}$ are $\Sigma_{1}^{1}$ subsets of $A$.

Proposition 7.6 (Covering Theorem) Suppose $A$ is $\Pi_{1}^{1}$ and $T$ is a tree such that

$$
f \in A \Longleftrightarrow T(f) \text { has no branches of length } \kappa .
$$

Suppose $B \subseteq A$ is $\Sigma_{1}^{1}$. Then there is $g \in \mathcal{T} \mathcal{O}$ such that $B \subseteq A^{T, g}$.

Proof. Let $S$ be a tree such that $f \in B$ if and only if $S(f)$ has a branch of length $\kappa$. Let $T^{\prime}$ be the tree of triples $(\bar{f}(\alpha), \bar{g}(\alpha), \bar{h}(\alpha))$ such that $\bar{g}(\alpha) \in T(f)$ and $\bar{h}(\alpha) \in S(f)$. If $T^{\prime}$ has a branch of length $\kappa$, then $f \in B \backslash A$. Let $T_{e} \cong T^{\prime}$. To prove $B \subseteq A^{T, e}$, let $f \in B$. Let $(\bar{h}(\alpha))_{\alpha<\kappa}$ be a branch of length $\kappa$ in $S(f)$. For $\bar{g}(\alpha) \in T(f)$ let $\phi(\bar{g}(\alpha))=(\bar{f}(\alpha), \bar{g}(\alpha), \bar{h}(\alpha))$. Now $\phi: T(f) \rightarrow T^{\prime}$ is order-preserving, whence $f \in A^{T, e}$.

Corollary 7.7 (Boundedness Theorem) Suppose $A$ is $\Pi_{1}^{1}$ and $T$ is a tree such that

$$
f \in A \Longleftrightarrow T(f) \text { has no branches of length } \kappa .
$$

Then $A$ is $\Delta_{1}^{1}$ if and only if there is $g \in \mathcal{T O}$ such that $\forall f \in A\left(T(f) \leq T_{g}\right)$.

We finish the section by showing how these ideas and the concept of chain isomorphism join to give us at strong limit singular cardinals an analogue of the cornerstone of the analysis of countable models using descriptive set theory. Recall that we have fixed a cofinal $\kappa$-sequence $\vec{\lambda}=\left\langle\lambda_{\xi}: \xi<\kappa\right\rangle$ in $\lambda$. We shall only consider chain models with $\lambda$ as the universe and a decomposition $\left\langle A_{\alpha}: \alpha<\kappa\right\rangle$ such that every $A_{\alpha}$ is a subset of some $\lambda_{\beta}$, and vice verse, every $\lambda_{\beta}$ is a subset of some $A_{\alpha}$. Let us call these $\vec{\lambda}$-chain models. Recall that we have fixed a bijection $H:{ }^{\kappa} \mathcal{P}(\lambda) \rightarrow{ }^{\kappa} \lambda$. If $A$ is a $\vec{\lambda}$-chain model with decomposition $\bar{A}=\left\langle A_{\alpha}: \alpha<\kappa\right\rangle$, then and $\langle A\rangle \frown \bar{A}$ is in the domain of $H$ and it is mapped to some $f \in{ }^{\kappa} \lambda$. We say that $A$ is coded by $f$. The orbit of $A$ is the set of $g \in{ }^{\kappa} \lambda$ which code some $B$ (and a decomposition of it) chain-isomorphic to $A$. The orbit depends on the function $H$.

Theorem 7.8 Suppose that $\lambda$ is a singular strong limit cardinal of cofinality $\kappa$. Then the orbit of a $\vec{\lambda}$-chain model $A$ of cardinality $\lambda$ and length $\kappa$ is a $\Sigma_{1}^{1}$-set in the space ${ }^{\kappa} \lambda$ equipped with the above topology. The orbit is $\Delta_{1}^{1}$ iff there is a $(\lambda, \kappa)$-Tree T such that for any chain model $B$ of cardinality $\lambda$ and length $\kappa$, I has a winning strategy in $E F D_{T}^{c,<\lambda}(A, B)$ if and only if $A \neq_{\text {chain }} B$. 
Proof. Suppose $A$ is a $\vec{\lambda}$-chain model with decomposition $\left\langle A_{\alpha}: \alpha<\kappa\right\rangle$. Let $\tau$ be a strategy of $\mathrm{I}$ in $E F_{\kappa}^{c,<\lambda}(A, B)$ in which the $\alpha$ th move is for $\alpha=\nu+2 n, \nu=\cup \nu$, a sequence that enumerates $\lambda_{\nu+n} \backslash \bigcup_{\beta<\nu+n} \lambda_{\beta}$ in $A$, and for $\alpha=\nu+2 n+1, \nu=\cup \nu$, a sequence that enumerates $\lambda_{\nu+n} \backslash \bigcup_{\beta<\nu+n} \lambda_{\beta}$ in $B$. Note that the strategy is independent of $B$. For any $\vec{\lambda}$-chain model $B$ with some decomposition $\left\langle B_{\alpha}: \alpha<\kappa\right\rangle$ let $S(B)$ be the tree of plays in which I has used $\tau$ and II has played but has not lost yet. Then for all $B$,

$$
A \varlimsup_{\text {chain }} B \Longleftrightarrow S(B) \text { has no branches of length } \kappa \text {. }
$$

This set if $\Pi_{1}^{1}$ by Proposition 7.5, so the orbit of $A$, which is the complement of this set, is $\Sigma_{1}^{1}$.

If the set $\left\{B: A \cong_{\text {chain }} B\right\}$ is $\Delta_{1}^{1}$, then we can apply Boundedness Theorem to its complement. Therefore there is a tree $T$ of cardinality $\lambda$ without branches of length $\kappa$ such that

$$
A \varlimsup_{\text {chain }} B \text { implies } S(B) \leq T \text {. }
$$

We can now show that

$$
A \varlimsup_{\text {chain }} B \text { iff player I has a winning strategy in } E F D_{\sigma T}^{c,<\lambda}(A, B) \text { : }
$$

Suppose $A \approx_{\text {chain }} B$. Then $S(B) \leq T$. Hence I wins $E F D_{\sigma T}^{c,<\lambda}(A, B)$ with the strategy $\tau$. On the other hand, if $A \cong_{\text {chain }} B$, then II wins $E F_{\kappa}^{c,<\lambda}(A, B)$, so I cannot win $E F D_{\sigma T}^{c,<\lambda}(A, B) \cdot \star_{7.8}$ 
If $\lambda=\kappa=\omega$, then every orbit is $\Delta_{1}^{1}$ and the Scott height of $A$ is - essentially - the tree $T$ of the above Theorem. So Theorem 7.8 generalizes the Scott analysis of countable models to chain models of singular strong limit cardinality.

\section{References}

[Eh] A. Ehrenfeucht, An application of games to the completeness problem for formalized theories, Fundamenta Mathematicae, vol. 49, pg. 129-141, (1961).

[ChKe] C. C. Chang and H. J. Keisler, Model theory, North-Holland, 1st edition 1973, latest edition (1990).

[Fr1] R. Fraïssé, Sur une nouvelle classification des systémes de relations, Comptes Rendus (Mathématique), vol. 230 pg. 10221024 (1950).

[Fr2] R. Fraïssé, Sur quelques classifications des systìes de relations, Ph.D. thesis, University of Paris (1953); published in Publications Scientifiques de l'Université d'Alger, sér. A 1, pg. 35-182, (1954).

[GoHr] R. Gostanian and K. Hrbaček, , On the Failure of the Weak Beth Property, Proceedings of the American Mathematical Society, vol. 58, no. 1 pg. 245-249, (1976).

[HyVä] T. Hyttinen and J. Väänänen On Scott and Karp trees of uncountable models, Journal for Symbolic Logic vol. 55 no. 3, pg. 897-908, (1990).

[Ka] C. Karp, Infinite-quantifier languages and $\omega$-chains of models, in the Proceedings of the Tarski Symposium (Proc. Sympos. Pure Math., Vol. XXV, Univ. California, Berkeley, Calif., 1971), pg. 225-232. Amer. Math. Soc., Providence, R.I., (1974).

[KuTa] C. Kuratowski and A. Tarski, Les opérations logiques et les ensembles projectifs, Fundamenta Mathematicae, vol. 17, pg. 240-248, (1931).

[LuSi] N. N. Lusin and W. Sierpinski, Sur un ensemble non mesurable B Journal de Mathématiques Pures et Appliquées, vol. 2 sér 9, pg. 5372, (1923).

[Mak] M. Makkai, On the model theory of denumerably long formulas with finite strings of quantifiers, Journal of Symbolic Logic, vol. 34, pg. 437459, (1969).

[Mal] J. Malitz, Infinitary analogs of theorems from first order model theory Journal of Symbolic Logic, vol. 36, pg. 216228 (1971). 
[MeVä] A. Mekler and J. Väänänen Trees and $\Pi_{1}^{1}$-subsets of ${ }^{\omega_{1}} \omega_{1}$, Journal of Symbolic Logic, vol. 58 (3), pg. 1052-1070, (1993).

[NaSt] M. Nadel and J. Stavi, $L_{\infty \lambda}$-equivalence, isomorphism and potential isomorphism, Transactions of the American Mathematical Society, vol. 236, pg. 51-74, (1978).

[Oi] J. Oikkonen Chain models and infinitely deep languages, Preprint 13, University of Mathematics, Department of Mathematics, http://mathstat.helsinki.fi/reports/INDEX, 45 pg, (1991).

[Sc] D. Scott, Logic with denumerably long formulas and finite strings of quantifiers in Theory of Models (Proc. 1963 Internat. Sympos. Berkeley), pg. 329-341, NorthHolland, Amsterdam, (1965).

[ToVä] S. Todorčević and J. Väänänen, Trees and Ehrenfeucht-Frässé games, Annals of Pure and Applied Logic vol. 100, pg. 69-97, (1999).

[Vä1] J. Väänänen, The Craig Interpolation Theorem in abstract model theory, Synthese, vol. 164, pg. 401-420 (2008).

[Vä2] J. Väänänen, Models and Games, Cambridge University Press, to appear. 